This item was submitted to Loughborough's Research Repository by the author.

Items in Figshare are protected by copyright, with all rights reserved, unless otherwise indicated.

\title{
Self-similar solutions of certain coupled integrable systems
}

PLEASE CITE THE PUBLISHED VERSION

LICENCE

CC BY-NC-ND 4.0

\section{REPOSITORY RECORD}

Chakravarty, S., R.G. Halburd, and S.L. Kent. 2019. "Self-similar Solutions of Certain Coupled Integrable Systems”. figshare. https://hdl.handle.net/2134/466. 


\title{
Self-similar solutions of certain coupled integrable systems
}

\author{
S. Chakravarty*, R. G. Halburd ${ }^{* *}$ and S. L. Kent ${ }^{\dagger}$ \\ * Department of Mathematics, University of Colorado, Colorado Springs, CO 80933-7150 \\ ** Department of Mathematical Sciences, Loughborough University, Loughborough, LE11 3TU, UK \\ $\dagger$ Department of Mathematics \& Statistics, Youngstown State University, Youngstown, OH 44555
}

September 6, 2002

\begin{abstract}
Similarity reductions of the coupled nonlinear Schrödinger equation and an integrable version of the coupled Maxwell-Bloch system are obtained by applying non-translational symmetries. The reduced system of coupled ordinary differential equations are solved in terms of Painlevé transcendents, leading to new exact self-similar solutions for these integrable equations.
\end{abstract}

PACS numbers: 02.30.Ik., 02.30.Hq., 02.30.Gp. 


\section{Introduction}

Completely integrable systems play an important role in many physical applications including water waves, plasma physics, field theory and nonlinear optics. An important feature of many integrable evolution equations is that a large class of their exact solutions, particularly the solitons, can be derived by applying the method of inverse scattering transform (IST) in appropriate variables (see $[1,2]$ for a review). Another significant characteristic shared by many (perhaps all) integrable partial differential equations (PDEs) is that their dimensional reductions to ordinary differential equations (ODEs) have solutions with no movable critical points. This remarkable property is known as the Painlevé property and the corresponding ODEs are said to be of "Painlevé type". The relationship between integrability and Painlevé property motivated Ablowitz, Ramani and Segur [3] to make the conjecture (hereafter referred to as the ARS conjecture) that a nonlinear evolution equation is solvable by IST only if every ODE obtained by exact similarity reduction is of Painlevé type, perhaps after a transformation of variables. There is considerable evidence that the ARS conjecture is true despite the absence of a complete proof at the present time. Nonetheless, this conjecture provides a preliminary test that is useful to determine whether a PDE is integrable. Conversely, failure of the conjecture in a certain case would strongly suggest that the given PDE is not solvable via IST. Besides playing an important role in the identification of integrable PDEs, the dimensional reduction of PDEs also provide an effective method of obtaining special classes of exact solutions. Indeed a large number of self-similar solutions have been found for equations that are solvable by IST by applying the dimensional reduction technique. Typically, these solutions are expressible in terms of elliptic functions, Painlevé transcendents or their degenerations (see e.g. [2] and references therein). The similarity reduction method can even be applied to obtain special solutions of non-integrable equations.

In this article, we study the dimensional reductions of certain completely integrable equations which have important applications in nonlinear optics. The equations considered here are the two-component generalizations of the Maxwell-Bloch (MB) and the nonlinear Schrödinger equations. In the text, they are referred to as the coupled Maxwell-Bloch (CMB) equations describing the propagation of ultra short laser pulses in a resonant medium of three-level atoms, and the coupled nonlinear Schrödinger (CNLS) equations modeling optical pulses in a birefringent optical fiber supporting two linearly polarized propagation modes. The traveling wave and $\mathrm{N}$-soliton solutions obtained by IST and Bäcklund transformations have already been discussed in the literature for both the CMB $[4,5]$ and the CNLS $[6,7,8]$ equations. In this work, we construct new self-similar solutions of the CMB and CNLS equations obtained by imposing non-translational symmetries on the original PDEs. It is possible that these new solutions may be of interest in future applications such as optical systems with memory [9]. Moreover, like the original MB and NLS equations, the obtained self-similar solutions of the reduced CMB and CNLS equations are also found to be Painlevé type and are in fact given in terms of the Painlevé transcendents. But in spite of the similarity in the analysis of the Lie-point symmetries, between the coupled systems and the scalar MB and NLS equations, the solution process is more involved in the coupled case because of the increased number of degrees of freedom.

The paper has two main sections. In section 2, we consider the MB and the CMB equations. After introducing the requisite mathematical framework for these equations, we discuss their scaling reductions. The MB equations are treated first as a special case of the CMB equations. We show that the reduced system of ODEs can be interpreted as a Hamiltonian dynamical 
system with a time-dependent Hamiltonian. Then these reduced ODEs for both the MB and CMB equations are explicitly solved in terms of the third Painleve transcendent PIII. The similarity reductions of the NLS and the CNLS equations are discussed next in section 3 . These equations admit two types of non-translational symmetries, namely Galilean boost and scaling. For both the NLS and CNLS equations, the invariant solutions are found in terms of the second Painlevé transcendent PII in the case of Galilean boost, whereas for the scaling symmetry the corresponding invariant solutions are described by the fourth Painlevé transcendent PIV.

\section{The coupled Maxwell-Bloch equations}

The Maxwell-Bloch (MB) equations describe the propagation of ultra short optical pulses in a coherent medium of two-level atoms and arise in the study of self-induced transparency [10]. In the lossless case, the MB equations are integrable via the IST method even when the inhomogeneous broadening of the medium is taken into account [11]. In recent years, there also have been considerable theoretical and experimental interests in the propagation of a pair of matched pulses through an absorbing medium of three-level atoms [12, 13, 14]. These studies have applications in quantum coherence and interference phenomena (generated by two photon transitions) such as electromagnetically induced transparency [15], lasing with inversion [16] and production of high refractive index materials [17].

\subsection{Mathematical background}

In the slowly varying amplitude and lossless approximations, the spatio-temporal dynamics of a pulse pair in a three-level resonant medium is given by $[12,13,5]$ the Schrödinger equations

$$
\frac{\partial a_{1}}{\partial t}=i \Omega_{1} a_{3}, \quad \frac{\partial a_{2}}{\partial t}=i \Omega_{2} a_{3}, \quad \frac{\partial a_{3}}{\partial t}=i\left(\bar{\Omega}_{1} a_{1}+\bar{\Omega}_{2} a_{2}\right)
$$

and the Maxwell's equations

$$
\frac{\partial \Omega_{j}}{\partial z}+\frac{1}{v} \frac{\partial \Omega_{j}}{\partial t}=-2 i \mu_{j} a_{j} \bar{a}_{3}, \quad j=1,2 .
$$

In the above equations, $a_{k}$ is the probability amplitude of the atomic level $|k\rangle, k=1,2,3$, and $\Omega_{j}, j=1,2$ denote the (normalized) complex electromagnetic field amplitudes also called the Rabi frequencies corresponding to $|j\rangle-|3\rangle$ transitions. Here and throughout the rest of this paper, overbar indicates complex conjugate. Furthermore, only one-dimensional pulse propagation is considered in equation $2.1 \mathrm{~b}$ where both pulses are traveling along the $z$-direction with velocity $v$, and where $\mu_{j}, j=1,2$ are the propagation coefficients assumed to be equal (i.e., $\left.\mu_{1}=\mu_{2}=\mu \neq 0\right)$. For three-level systems, there are only two known integrable cases which correspond to the $\Lambda$ and $V$ configurations of the atomic energy levels. In above, $|3\rangle$ is the ground state for the $V$ system whereas for the $\Lambda$ system $|3\rangle$ corresponds to the highest excited state.

It is useful to introduce pulse-local coordinates: $\tau=z, x=t-z / v$, and to represent the Schrödinger-Maxwell equations (2.1a, 2.1b) in the form

$$
U_{\tau}=[\rho, J], \quad \rho_{x}=[U, \rho]
$$


where subscripts denote partial derivatives, and the $3 \times 3$ matrices $U, \rho$ and $J$ are defined as

$$
\begin{aligned}
& U \equiv\left(\begin{array}{ccc}
0 & 0 & u_{1} \\
0 & 0 & u_{2} \\
-\bar{u}_{1} & -\bar{u}_{2} & 0
\end{array}\right), \quad u_{j}=i \Omega_{j}, j=1,2, \\
& J \equiv \operatorname{diag}(-\mu,-\mu, \mu), \quad[\rho]_{i j} \equiv a_{i} \bar{a}_{j} .
\end{aligned}
$$

$\rho$ is the probability density matrix and has vanishing determinant. The second equation in (2.2a) for the matrix elements of $\rho$ are called the Bloch equations. We refer to equations (2.2a) and $(2.2 \mathrm{~b})$ as the coupled Maxwell-Bloch (CMB) equations. When $a_{1}=u_{1}=0$ (or $a_{2}=u_{2}=0$ ) in (2.2b), the CMB equations reduce to the MB equations in a medium of two-level atoms.

The system of equations (2.2a) can be expressed as the integrability condition of an associated linear system (Lax pair)

$$
\Psi_{x}=(U+\lambda J) \Psi, \quad \Psi_{\tau}=\frac{\rho}{\lambda} \Psi
$$

where $\lambda$ is the spectral parameter. The Lax-pair and the zero-curvature representation were exploited to obtain special classes of solutions of the CMB equation via IST and Bäcklund transformation techniques (see e.g. $[4,5]$ and references therein). A field theoretic description of the CMB equation in terms of symmetric spaces $S U(3) / U(2)$ was given in Ref. [18] where the hidden symmetries and conservation laws were also discussed. We remark that the MB and the CMB equations can also be obtained as dimensional reductions of the self-dual Yang-Mills equation with gauge groups $S U(2)$ and $S U(3)$, respectively. (Reductions of the Lax pair for the self-dual Yang-Mills equations giving rise to the above linear system for $\Psi$ was discussed for example, in Ref. [19]). In this article, our aim is to study the similarity reductions of the CMB equations and to derive its self-similar solutions that are distinct from the traveling wave or breather type solutions obtained earlier [5]. Furthermore, we wish to investigate whether these self-similar solutions possess the Painlevé property which is a characteristic feature of integrable equations. We first consider the similarity reduction of the MB system which is a special case of the $\mathrm{CMB}$ equation, and treat the more general case next.

\subsection{Reduction of the Maxwell-Bloch system}

If we set $a_{1}=\Omega_{1}=0$ (or $a_{2}=\Omega_{2}=0$ ) in equations (2.1a, 2.1b), then this leads to the Schrödinger-Maxwell equations for a two-level medium. As mentioned above, the corresponding $\mathrm{CMB}$ equations then reduce to the MB equations. The resulting equations $(2.2 \mathrm{a})$ and $(2.2 \mathrm{~b})$ (with $a_{2}=u_{2}=0$ ) can be expressed in the form

$$
\begin{aligned}
& \hat{U}_{\tau}=[\hat{\rho}, \hat{J}], \quad \hat{\rho}_{x}=[\hat{U}, \hat{\rho}], \\
& \hat{U} \equiv\left(\begin{array}{cc}
0 & u \\
-\bar{u} & 0
\end{array}\right), \quad \hat{J} \equiv \operatorname{diag}(-\mu, \mu), \quad[\hat{\rho}]_{i j} \equiv a_{i} \bar{a}_{j}, i, j=1,3,
\end{aligned}
$$

and describe the propagation of a single pulse in a two-level resonant medium in the absence of inhomogeneous broadening (sharp line limit). Here we point out that equations (2.3) differ slightly from the usual form of MB equations which appear in the literature (see e.g., [11]) where the trace-free part of $\hat{\rho}$ namely, $\hat{\rho}_{0} \equiv \hat{\rho}-\operatorname{Tr}(\hat{\rho}) / 2$ is used instead of $\hat{\rho}$ itself. However since the trace satisfies $\operatorname{Tr}(\hat{\rho})_{x}=0$ (which leads to the conservation of probability density with suitable initial conditions), it follows that (2.3) is equivalent to the MB equations with $\hat{\rho}$ replaced by $\hat{\rho}_{0}$. 
It is also worth noting that if the field $u(x, 0)$ is real (or has constant phase), then $u(x, \tau)$ stays real (or its phase remains constant). In this case, equation (2.3) is equivalent to the sine-Gordon equations which has similarity reductions to the third Painlevé equation (see e.g., [2]).

A class of self-similar solutions of the MB equations arise from the investigation of Lie-point symmetries which leave equation (2.3) invariant. Besides the translational symmetries which lead to traveling wave solutions, the MB equations admit a 1-parameter subgroup of scaling symmetries: $x \rightarrow \varepsilon^{-1} x, \tau \rightarrow \varepsilon \tau, \hat{U} \rightarrow \varepsilon \hat{U}$ and $\hat{\rho} \rightarrow \hat{\rho}$. The solutions invariant under the scaling symmetry have the form

$$
\hat{U}(x, \tau)=\hat{Q}(\xi) / x, \quad \hat{\rho}(x, \tau)=\hat{\rho}(\xi), \quad \xi=\sqrt{x \tau} .
$$

Substituting the above form of $\hat{U}$ and $\hat{\rho}$ into equation (2.3) yields the following set of coupled ODEs for the off-diagonal matrix elements $q, \bar{q}$ of $\hat{Q}$ and $\hat{\rho}_{i j}$

$$
\begin{aligned}
& q^{\prime}=4 \xi \mu \hat{\rho}_{13}, \quad \xi \hat{\rho}_{13}{ }^{\prime}=2 q\left(\hat{\rho}_{33}-\hat{\rho}_{11}\right), \\
& \xi \hat{\rho}_{11}{ }^{\prime}=-\xi \hat{\rho}_{33}{ }^{\prime}=2\left(q \hat{\rho}_{31}+\bar{q} \hat{\rho}_{13}\right), \quad \hat{\rho}_{31} \equiv \overline{\hat{\rho}_{13}},
\end{aligned}
$$

where prime indicates derivative with respect to the argument. The system of ODEs (2.4) admit the first integrals

$$
\hat{\rho}_{11}+\hat{\rho}_{33}=\operatorname{Tr}(\hat{\rho})=a, \quad q \hat{\rho}_{31}-\bar{q} \hat{\rho}_{13}=\operatorname{Tr}(\hat{Q} \hat{\rho})=b,
$$

where $a$ and $b$ are constants and $a \neq 0$. Making use of the first integrals and the fact that $\operatorname{det} \hat{\rho}=\hat{\rho}_{11} \hat{\rho}_{33}-\hat{\rho}_{13} \hat{\rho}_{31}=0$, it is possible to reduce (2.4) to a system of two first order ODEs

$$
\begin{aligned}
& y^{\prime}-4 \mu+\frac{y}{\xi}=2 y^{2}\left(\hat{\rho}_{11}-\hat{\rho}_{33}\right), \quad \text { where } \quad y=\frac{q}{\hat{\rho}_{13} \xi} \\
& \left(\hat{\rho}_{11}-\hat{\rho}_{33}\right)^{\prime}=2 y\left[a^{2}-\left(\hat{\rho}_{11}-\hat{\rho}_{33}\right)^{2}\right]-\frac{4 b}{\xi} .
\end{aligned}
$$

If we eliminate the quantity $\hat{\rho}_{11}-\hat{\rho}_{33}$ from the equations immediately above and make the change of variables

$$
W=\lambda y, \quad Z=k \xi, \quad \text { such that } \quad \lambda k=a \quad \text { and } \quad k \lambda^{-1}=2 \mu,
$$

in the resulting second order ODE for $y$, then we obtain the third Painlevé equation PIII for $W(Z)[20]$ (p. 335, equation XIII)

$$
\frac{d^{2} W}{d Z^{2}}=\frac{1}{W}\left(\frac{d W}{d Z}\right)^{2}-\frac{1}{Z}\left(\frac{d W}{d Z}\right)+\frac{\alpha W^{2}+\beta}{Z}+\gamma W^{3}+\frac{\delta}{W}
$$

with the choice of parameters $\alpha=-8 b / a$ and $\beta=\gamma=-\delta=4$. Note that in the present context $W(z)$ represents the solution of a 1-parameter family of PIII transcendents since 3 of the 4 parameters are fixed. (In general, only 2 of the 4 parameters in PIII are free, the remaining 2 parameters can be fixed by suitable re-scalings of the dependent and independent variables). Finally, working backward from (2.6) it is straightforward to show that the scale invariant solutions of the MB equations given by equation (2.4) can be expressed in terms of $W(Z)$ and $W^{\prime}(Z)$. We omit the details.

It is also possible to derive the PIII reduction of the MB equations by a slightly different route which actually leads to the fifth Painlevé equation PV with special parameter values. This 
special PV equation can be reduced to PIII by a known transformation [21]. We outline this approach here since it is also relevant to the similarity reduction of the CMB equations to be discussed next. If we use the variable $\hat{g} \equiv \hat{\rho}_{11}$ instead of $y$, then by differentiating the equation for $\hat{\rho}_{11}$ in (2.4) we get

$$
\hat{g}^{\prime \prime}=\left(\frac{\hat{g}^{\prime 2}}{2}-\frac{2 b^{2}}{\xi^{2}}\right)\left(\frac{1}{\hat{g}}-\frac{1}{a-\hat{g}}\right)-\frac{\hat{g}^{\prime}}{\xi}+16 \mu \hat{g}(a-\hat{g}) .
$$

To derive the above equation for $\hat{g}$, we used the remaining equations from (2.4), the first integrals from (2.5) and the relation $\hat{\rho}_{11} \hat{\rho}_{33}=\hat{\rho}_{13} \hat{\rho}_{31}$ (i.e., $\operatorname{det} \hat{\rho}=0$ ). Next, by introducing the variable $\hat{y}=(\hat{g}-a) / \hat{g}$ we can further transform the above ODE to

$$
\hat{y}^{\prime \prime}=\left(\frac{1}{2 \hat{y}}+\frac{1}{\hat{y}-1}\right) \hat{y}^{\prime 2}-\frac{\hat{y}^{\prime}}{\xi}+\frac{4(\hat{y}-1)^{2}}{\xi^{2}}\left(\alpha \hat{y}+\frac{\beta}{y}\right)+2 \gamma \hat{y},
$$

where $\alpha=b^{2} / 2 a^{2}=-\beta$ and $\gamma=-8 \mu a$. It is equation (2.7) that can either be transformed to a special PV equation with 2 free parameters or to a special PIII equation with 1 free parameter, thereby inducing a transformation between the special cases of PIII and PV themselves. We defer the details of these transformations to the next subsection where they will be discussed in a more general context.

\subsection{Reduction of the coupled Maxwell Bloch equations}

Like the MB equations, the CMB equations also admit ODE reductions under the scaling symmetry. The scaling invariant solutions are obtained from (2.2a) by expressing the $3 \times 3$ matrices in $(2.2 \mathrm{~b})$ in the form

$$
U(x, \tau)=Q(\xi) / x, \quad \rho(x, \tau)=\rho(\xi), \quad \xi=\sqrt{x \tau},
$$

where the matrices $Q(\xi)$ and $\rho(\xi)$ satisfy

$$
\xi \rho^{\prime}=2[Q, \rho], \quad Q^{\prime}=2 \xi[\rho, J] .
$$

Alternatively, one can directly consider the scaling reductions of the Schrödinger-Maxwell equations $(2.1 \mathrm{a}, 2.1 \mathrm{~b})$ by expressing the electromagnetic fields and the probability amplitudes as

$$
i \Omega_{j}(z, t)=u_{j}(x, \tau)=q_{j}(\xi) / x, j=1,2 \quad a_{k}(z, t)=a_{k}(x, \tau)=a_{k}(\xi), k=1,2,3 .
$$

The reduced system of ODEs are simpler to express in terms of the variables $a_{k}$ rather than the Bloch matrix-elements $\rho_{i j}=a_{i} \bar{a}_{j}$ (which are quadratic in the $a_{k}$ ). The ODEs for the variables $q_{j}(\xi), j=1,2$ and $a_{k}(\xi), k=1,2,3$ are given by

$$
q_{j}{ }^{\prime}=4 \xi \mu a_{j} \bar{a}_{3}, \quad \xi a_{j}{ }^{\prime}=2 q_{j} a_{3}, j=1,2, \quad \xi a_{3}{ }^{\prime}=-2\left(\bar{q}_{1} a_{1}+\bar{q}_{2} a_{2}\right) .
$$

Equation (2.9) together with the complex conjugate equations imply the matrix ODE system (2.8). The reduced ODEs admit the following first integrals

$$
\begin{aligned}
& \sum_{k=1}^{3}\left|a_{k}\right|^{2}=\operatorname{Tr}(\rho)=A, \quad \sum_{j=1}^{2}\left(a_{3} q_{j} \bar{a}_{j}-c . c .\right)=\operatorname{Tr}(Q \rho)=B, \\
& q_{2} a_{1}-q_{1} a_{2}=C, \quad\left(|C|^{2}=\operatorname{Tr}\left(\rho Q^{2}-A Q^{2} / 2\right)\right),
\end{aligned}
$$


where $A, B, C$ are constants and c.c. denotes complex conjugate. Note that unlike the reduced MB equations (2.4), the reduced CMB equations (2.9) admit an additional first integral $C(C \equiv 0$ for the MB equations).

It is interesting to note that the equations (2.9) and their complex conjugates can be recast as a Hamiltonian dynamical system on a 10-dimensional (real) phase space with variables $q_{j}, j=$ 1,2 and $b_{k} \equiv \xi a_{k}, k=1,2,3$ together with their complex conjugates. When expressed in terms of the new variables $b_{k}$, equation (2.9) becomes

$$
\xi q_{j}{ }^{\prime}=4 \mu b_{j} \bar{b}_{3}, \quad \xi b_{j}{ }^{\prime}=b_{j}+2 q_{j} b_{3}, j=1,2, \quad \xi b_{3}{ }^{\prime}=b_{3}-2\left(\bar{q}_{1} b_{1}+\bar{q}_{2} b_{2}\right) .
$$

Equation $\left(2.9^{\prime}\right)$ represents a Hamiltonian system prescribed by the canonical Poisson bracket relations and a time $(\xi)$-dependent Hamiltonian function $H$ as follows

$$
\begin{aligned}
q_{j}{ }^{\prime} & =\left\{q_{j}, H\right\}, \quad j=1,2, \quad b_{k}{ }^{\prime}=\left\{b_{k}, H\right\}, \quad k=1,2,3, \\
H & =\frac{1}{\xi}\left(\sum_{k=1}^{3}\left|b_{k}\right|^{2}+2 \sum_{j=1}^{2}\left(b_{3} q_{j} \bar{b}_{j}-\text { c.c. }\right)\right)=\xi \operatorname{Tr}(\rho+2 Q \rho) .
\end{aligned}
$$

The associated Poisson structure is defined by the fundamental Poisson brackets

$$
\left\{q_{i}, \bar{q}_{j}\right\}=-2 \mu \delta_{i j}, \quad i, j=1,2 \quad\left\{a_{k}, \bar{a}_{l}\right\}=\delta_{k l}, \quad k, l=1,2,3,
$$

where $\delta_{i j}$ is the usual Kronecker delta, and all other fundamental Poisson brackets vanish. Furthermore, the first integrals in equation (2.10) are in involution with respect to the Poisson structure defined above.

The next task is to solve the system (2.9) and to determine whether the general solution has the Painlevé property. The similarity reductions of the CMB equations are expected to be of Painlevé type because of the ARS conjecture. However to our knowledge, the Painlevé property of these ODEs have not yet been studied. In what follows, we show that there exists a suitable combination of variables (not necessarily unique) that satisfies the third Painlevé equation PIII in general position. Secondly, the CMB variables in (2.9) can be expressed via the PIII transcendent either by quadratures or through the solution of a Riccati equation.

It is useful for subsequent calculations to introduce the variables:

$$
g \equiv\left|a_{1}\right|^{2}+\left|a_{2}\right|^{2}=A-\left|a_{3}\right|^{2} \quad \text { and } \quad f \equiv a_{3}\left(\bar{a}_{1} q_{1}+\bar{a}_{2} q_{2}\right) .
$$

Note that the first integral $B$ in equation (2.10) can be written as $B=f-\bar{f}$. Starting from equation (2.9) and making use of the first integrals (2.10), a straightforward computation yields

$$
\begin{array}{ll} 
& \xi g^{\prime}=4 f-2 B, \quad \xi f^{\prime}=4 \mu \xi^{2} g(A-g)+2 h(A-2 g)+2|C|^{2} \\
\text { where } & h \equiv\left|q_{1}\right|^{2}+\left|q_{2}\right|^{2}=\frac{f(f-B)}{g(A-g)}+\frac{|C|^{2}}{g} .
\end{array}
$$

Eliminating $f(\xi)$ from the two first order equations (2.12), we obtain

$$
g^{\prime \prime}=\left(\frac{g^{\prime 2}}{2}-\frac{2 B^{2}}{\xi^{2}}\right)\left(\frac{1}{g}-\frac{1}{A-g}\right)-\frac{g^{\prime}}{\xi}+\frac{8|C|^{2}}{\xi^{2}}\left(\frac{A-g}{g}\right)+16 \mu g(A-g)
$$

which is rational in $g(\xi)$ and $g^{\prime}(\xi)$, and is of Painlevé type. Indeed, by the rational substitution $y=(g-A) / g$, the above ODE for $g(\xi)$ can be transformed into the following intermediate form

$$
y^{\prime \prime}=\left(\frac{1}{2 y}+\frac{1}{y-1}\right) y^{\prime 2}-\frac{y^{\prime}}{\xi}+\frac{4(y-1)^{2}}{\xi^{2}}\left(\alpha y+\frac{\beta}{y}\right)+2 \gamma y,
$$


where $\alpha=\left(B^{2}-4 A|C|^{2}\right) / 2 A^{2}, \beta=-B^{2} / 2 A^{2}$ and $\gamma=-8 \mu A$. Here we note that when $C=0$, equation (2.13) reduces to equation (2.7) of the previous subsection. This corresponds to the special cases of the CMB equations when either (a) $q_{1} / q_{2}=a_{1} / a_{2}, q_{j} \neq 0, a_{j} \neq 0, j=1,2$ or (b) $q_{j}=a_{j}=0, j=1$ or 2 . The latter case (b) is simply the degeneration of the CMB equations to the MB equations.

Changing the independent variable to $Z=\xi^{2} / 2$ in equation (2.13), leads to a special case of the fifth Painlevé equation PV [20] (p. 341, equation XXXIX, $\delta=0$ )

$$
\frac{d^{2} W}{d Z^{2}}=\left(\frac{1}{2 W}+\frac{1}{W-1}\right)\left(\frac{d W}{d Z}\right)^{2}-\frac{1}{Z} \frac{d W}{d Z}+\frac{(W-1)^{2}}{Z^{2}}\left(\alpha W+\frac{\beta}{W}\right)+\frac{\gamma W}{Z},
$$

with the same parameters $\alpha, \beta, \gamma$ as defined above. There also exists a correspondence between $y(\xi)$ in equation (2.13) and solutions of PIII in general position (i.e., with two free parameters). This transformation [21] between solutions of PIII and the special case of PV with $\delta=0$ is given as follows. Define the function $\widehat{W}(\xi)$ via the solution $y(\xi)$ of equation $(2.13)$ by

$$
\widehat{W}=-\frac{y^{\prime}}{2 y}+\frac{c_{1} y}{\xi}+\frac{c_{2}}{y \xi}-\frac{c_{1}+c_{2}}{\xi}
$$

where $c_{1}^{2}=2 \alpha$ and $c_{2}^{2}=-2 \beta$ are given in terms of the parameters $\alpha, \beta$ of equation (2.13). Then $\widehat{W}(\xi)$ is a solution of PIII and satisfies equation (2.6) with parameters $\hat{\alpha}, \hat{\beta}, \hat{\gamma}, \hat{\delta}$ where $\hat{\alpha}=2\left(c_{1}+c_{2}\right), \hat{\beta}=2\left(c_{1}-c_{2}-1\right) / \gamma, \hat{\gamma}=1$ and $\hat{\delta}=-\gamma^{2}$.

Thus we have shown how to express $y(\xi)$ or equivalently, $g(\xi)=\left|a_{1}\right|^{2}+\left|a_{2}\right|^{2}$ in terms of PV (or PIII) transcendent. In fact, it turns out that the solution of the full system of ODEs (2.9) can be obtained in terms of this Painlevé transcendent and its derivative, as outlined below. Given $g(\xi)$, we solve for $f(\xi)$ from the first equation in (2.12). Next, from (2.9) we obtain

$$
Y_{j}^{\prime}=2(g-A) Y_{j}^{2}-\frac{2 f Y_{j}}{g-A}+4 \xi \mu, \quad \text { where } \quad Y_{j}=\frac{q_{j}}{a_{j} \bar{a}_{3}}, j=1,2 \quad \text { and } \quad \frac{\bar{a}_{3}^{\prime}}{\bar{a}_{3}}=\frac{2 f}{g-A}
$$

The last equation above can be solved for $\bar{a}_{3}$. Then from two independent solutions $Y_{1}$ and $Y_{2}$ of the Riccati equation, the CMB variables $q_{j}$ and $a_{j}, j=1,2$ can be obtained in quadratures as follows:

$$
\frac{q_{j}^{\prime}}{q_{j}}=\frac{4 \xi \mu}{Y_{j}}, \quad a_{j}=\frac{q_{j}}{\bar{a}_{3} Y_{j}}, \quad j=1,2 .
$$

The remaining variables $\bar{q}_{j}, \bar{a}_{j}$ and $a_{3}$ can be obtained in a similar fashion, starting with $g(\xi)$ and $\bar{f}(\xi)=f(\xi)-B$. Thus all the CMB variables in (2.9) can be obtained in terms of $g$ and $f$ either by quadratures or by solving Riccati equations.

We conclude this section by remarking that the similarity reductions of the Maxwell-Bloch equations for two and three-level atomic media can be extended to the multi-level case of $n$-CMB equation describing $n$ electromagnetic fields propagating in a resonant medium with $n+1$ energy states for $n>2$. This can be achieved in a relatively straightforward manner by appropriate generalizations of the equations (2.8-2.10). Besides $\operatorname{Tr}(\rho)$ and $\operatorname{Tr}(Q \rho)$, there are $n(n-1) / 2$ additional first integrals given by $C_{i j}=q_{i} a_{j}-q_{j} a_{i}, i \neq j, i, j=1,2 \ldots n$. By introducing the variable $\tilde{g}(\xi) \equiv\left|a_{1}\right|^{2}+\left|a_{2}\right|^{2}+\ldots+\left|a_{n}\right|^{2}$ and proceeding in the same way as we did for the CMB reduction, the scaling invariant solutions of the $n$-CMB equations are also obtained via the general PIII transcendent. We do not go into any further details of the $n$-CMB case in this article. 


\section{The coupled nonlinear Schrödinger equations}

In this section we study the similarity reductions of a system of two coupled NLS (CNLS) equations in $1+1$ dimensions. This system is known to be completely integrable in the sense that it admits soliton solutions and the initial value problem can be solved via the IST method [6]. The CNLS equations also possess an infinite set of conserved quantities [7], and passes the Painlevé PDE test [8]. Thus it is reasonable to expect that in accord with the ARS conjecture, the ODEs obtained by the dimensional reductions of the CNLS equations are of Painlevé type. Indeed in Ref.[22], a set of Painlevé type ODEs regarded as a coupled system of Painlevé II equations was derived by reducing the CNLS by the Galilean boost symmetry. In this section we derive a more general set of coupled ODEs and show that their solutions are given directly via the second Painlevé transcendent. We also present another dimensional reduction of the CNLS using scaling symmetry and obtain the invariant solutions in terms of the fourth Painlevé transcendent.

\subsection{Reductions of NLS equations}

Here we review briefly the known similarity reductions of the NLS equation

$$
i u_{t}=\frac{u_{x x}}{2}+s|u|^{2} u,
$$

for the complex function $u(x, t)$. We consider both focusing $(s=1)$ as well as defocussing $(s=-1)$ cases. Analysis of the classical Lie-point symmetries of equation (3.1) leads to three types of similarity reductions (see e.g., [23]) namely, translation, scaling and Galilean boost. The translational symmetry leads to the well known traveling wave solutions. In this article, we discuss the non-translational symmetries only. There are two cases that we consider.

Case 1. Galilean boost ([23]): In this case the form of the field $u(x, t)$ is given by

$$
u(x, t)=r(z) \exp \left(i \phi(z)-i \alpha t\left(x-\alpha t^{2} / 3\right)\right), \quad z=x-\alpha t^{2} / 2
$$

where $\alpha>0$ is a constant parameter. Substituting (3.2) into equation (3.1) and separating real and imaginary parts, we find that $r(z)$ and $\phi(z)$ satisfy the system of equations

$$
\begin{aligned}
r \phi^{\prime \prime}+2 r^{\prime} \phi^{\prime} & =0 \\
r^{\prime \prime}-r \phi^{2}+2 s r^{3}-2 \alpha z r & =0 .
\end{aligned}
$$

Equation (3.3a) may be integrated once to obtain $\phi^{\prime}=C / r^{2}$, where $C$ is the constant of integration. Substituting the result into (3.3b) leads to a non-autonomous ODE for $r(z)$

$$
r^{\prime \prime}=C^{2} r^{-3}-2 s r^{3}+2 \alpha z r .
$$

If $C=0$, equation (3.4) can simply be rescaled to

$$
\frac{d^{2} W}{d Z^{2}}=2 W^{3}+Z W, \quad \text { where } \quad W=(-s)^{1 / 2}(2 \alpha)^{-1 / 3} r, \quad Z=(2 \alpha)^{1 / 3} z,
$$

which is a special case of the second Painlevé equation PII [20] (p. 334, equation IX with $\gamma=0$ ). When $C \neq 0$, equation (3.4) is still solvable in terms of a Painlevé type ODE. By an appropriate change of variables in equation (3.4), it is possible to derive the following equation

$$
\frac{d^{2} W}{d Z^{2}}=\frac{1}{2 W}\left(\frac{d W}{d Z}\right)^{2}+4 a W^{2}-Z W-\frac{1}{2 W},
$$


where,

$$
W=\frac{(4 \alpha)^{1 / 3}}{2 i C} r^{2}, \quad Z=-(4 \alpha)^{1 / 3} z, \text { and } \quad a= \pm \frac{i s C}{2 \alpha} .
$$

Equation (3.6) is also a Painlevé type ODE (PXXXIV) which can be transformed to PII (see e.g., [20] p. 340, equation XXXIV).

Case 2. Scaling reduction: The form of $u(x, t)$ is given by,

$$
u(x, t)=t^{(-1+i \mu) / 2} q(z), \quad z=x t^{-1 / 2},
$$

where the constant $\mu$ is real. Substituting (3.7) into (3.1) yields the following ODE for $q(z)$

$$
i q^{\prime \prime}=z q^{\prime}+q-i\left(\mu+2 s|q|^{2}\right) q .
$$

If one defines $q(z)=r(z) \exp (i \phi(z))$ and proceeds in the same way as in the case of the Galilean boost reduction, then it is possible to eventually obtain a third order ODE in the nonlocal variable $v(z)$, where $v^{\prime}(z)=r^{2}(z)$. The equation for $v(z)$ can be integrated once to obtain a second order, second degree ODE which possess the Painlevé property and whose solution can be expressed in terms of the PIV transcendent and its derivatives [24, 25]. Here we do not give the details of this reduction procedure which can be found in the literature (see, e.g., [26, 27]). Instead, we describe a different procedure to obtain the PIV equation more directly from (3.8) and in terms of a local variable defined in terms of $q(z)$ and its derivative. ¿From equation (3.8) and its complex conjugate, we construct a first order system of ODEs

$$
b^{\prime}=2 a q, \quad \bar{b}^{\prime}=-2 a \bar{q}, \quad a^{\prime}=s(q \bar{b}-\bar{q} b),
$$

where $b=-i q^{\prime}+z q, \bar{b}$ is the complex conjugate of $b$ and $2 a=i\left(\mu+2 s|q|^{2}\right)$. Note that the above system admits a first integral given by,

$$
a^{2}-s|b|^{2}=-m^{2}
$$

where $m$ is a constant. Next we introduce a new variable $y \equiv b / q=-i q^{\prime} / q+z$, which is local in $q(z)$ and $q^{\prime}(z)$ unlike the previously discussed similarity reduction. Then it is a straightforward calculation by using equations $(3.9 \mathrm{a}, 3.9 \mathrm{~b})$ to obtain a second order ODE for $y(z)$, namely,

$$
y^{\prime \prime}=\frac{y^{\prime 2}}{2 y}-\frac{3 y^{2}}{2}+2 z y^{2}+y\left(\mu+i-z^{2} / 2\right)+\frac{2 m^{2}}{y} .
$$

After a rescaling of the dependent and independent variables in equation (3.10)

$$
y=\beta W, \quad z=k Z, \quad \text { such that } \quad \beta=-k, \quad k^{4}=-4,
$$

we obtain equation PIV [20](p. 339, equation XXXI)

$$
\frac{d^{2} W}{d Z^{2}}=\frac{1}{2 W}\left(\frac{d W}{d Z}\right)^{2}-\frac{3 W^{2}}{2}+4 Z W^{2}+2\left(Z^{2}-c\right) W+\frac{8 m^{2}}{W}
$$

with $c= \pm(-1+i \mu)$. 


\subsection{Reductions of the CNLS equation}

Next we consider the similarity reductions of the CNLS equation

$$
i u_{j t}=\frac{u_{j x x}}{2}+s\left(\left|u_{1}\right|^{2}+\left|u_{2}\right|^{2}\right) u_{j}, \quad j=1,2
$$

for the pair of complex function $u_{j}(x, t)$, where $s=1$ and $s=-1$ are the focusing and defocussing cases, respectively. As in the previous subsection, we consider the two cases of Galilean boost and scaling symmetries.

Case 1. Galilean boost: The CNLS fields $u_{j}(x, t)$ and the similarity variable are expressed as

$$
u_{j}(x, t)=r_{j}(z) \exp \left(i \phi_{j}(z)-i \alpha t\left(x-\alpha t^{2} / 3\right)\right), \quad z=x-\alpha t^{2} / 2, \quad j=1,2,
$$

where $\alpha>0$ is a constant. Using the form (3.13) in equation (3.12), we obtain a set of coupled ODEs for the amplitude $r_{j}(z)$ and the phase $\phi_{j}(z)$ components

$$
\begin{aligned}
r_{j} \phi_{j}^{\prime \prime}+2 r_{j}^{\prime} \phi_{j}^{\prime} & =0 \\
r_{j}^{\prime \prime}-r_{j} \phi_{j}^{\prime}{ }^{2}+2 s\left(r_{1}^{2}+r_{2}^{2}\right) r_{j}-2 \alpha z r_{j} & =0, \quad j=1,2 .
\end{aligned}
$$

Once again, equation (3.14a) can be integrated to obtain $\phi_{j}^{\prime}=C_{j} / r_{j}^{2}$ where $C_{j}, j=1,2$ are constants of integration. Then equation $(3.14 \mathrm{~b})$ reduces to a coupled non-autonomous system for the $r_{j}$ given by

$$
r_{j}^{\prime \prime}=C_{j}^{2} r_{j}^{-3}+2(\alpha z-s g) r_{j}, \quad j=1,2,
$$

where we have defined $g(z) \equiv r_{1}^{2}(z)+r_{2}^{2}(z)$.

Equation (3.15) with $C_{j}=0$ can be regarded as a coupled system of PII equations in the variables $r_{1}$ and $r_{2}$. This system was derived in Ref.[22] where the authors performed a local analysis (in the neighborhood of some initial values) which suggested that (3.15) possess the Painlevé property. Here we show that (3.15) does indeed have the Painlevé property for any choice for the constants $C_{j}$ since equation (3.15) can be explicitly solved in terms of the PII transcendent. To that end, we re-express equation (3.15) in terms of the variables $y_{j}=r_{j}^{2}$ and differentiate the resulting equation once to arrive at

$$
y_{j}^{\prime \prime \prime}=8 y_{j}{ }^{\prime}(\alpha z-s g)+4 y_{j}\left(\alpha-s g^{\prime}\right), \quad j=1,2 .
$$

Now the basic idea is to regard equation (3.16) as a linear, third order equation for each component $y_{j}(z)$, with variable coefficients depending on $g(z)$ and $g^{\prime}(z)$. Thus the solution of equation (3.15) is completely expressible in terms of the solutions of the linear equation (3.16), provided the function $g(z)$ is known. An ODE for $g(z)$ can be found readily if we add the two equations for $y_{1}$ and $y_{2}$ in (3.16) and introduce the variable $h(z)$ where $h^{\prime}(z)=g(z)=y_{1}+y_{2}$. After integrating the resulting equation once, we obtain

$$
h^{\prime \prime \prime}+6 h^{\prime 2}-8 \alpha z h^{\prime}+4 \alpha h-\beta=0,
$$

where $\beta$ is the constant of integration. We remark at this point that the reduction procedure outlined thus far can be easily extended to the $n$-CNLS equation with complex fields $u_{j}, j=$ $1,2, \ldots n, n>2$. This is achieved by appropriately generalizing equations (3.12)-(3.16) for $n$ components and by defining the function $g(z)$ as $g(z) \equiv y_{1}+y_{2}+\ldots+y_{n}$.

Equation (3.17) is of Painlevé type and belongs to the third order, polynomial class of Painlevé type equations studied by Chazy [24] and later by Bureau [25]. To make the connection 
with PII, we note that equation (3.17) admits a first integral which is of second order and second degree, namely

$$
h^{\prime \prime 2}+4 h^{\prime 3}-4 \alpha z h^{\prime 2}+4 \alpha h h^{\prime}-\beta h^{\prime}=\gamma,
$$

where $\gamma$ is an integration constant. Two of the three constants $\alpha, \beta, \gamma$ can be absorbed by the following transformation of variables

$$
\sigma_{I I}=k\left(h+\beta k^{3} / 2\right), \quad Z=z / k, \quad k=-(2 \alpha)^{-1 / 3}
$$

in the above equation which then takes the form

$$
\left(\frac{d \sigma_{I I}}{d Z}\right)^{2}+4\left(\frac{d \sigma_{I I}}{d Z}\right)^{3}+2 Z\left(\frac{d \sigma_{I I}}{d Z}\right)^{2}-2 \sigma_{I I} \frac{d \sigma_{I I}}{d Z}-\gamma=0 .
$$

The transformation of equation (3.18) into the PII equation was discussed in Refs. [24, 25]. More recently, it was noted in Ref. [28] that $\sigma_{I I}(Z)$ satisfying equation (3.18), is the Hamiltonian function for PII, and is also related to the $\tau$-function simply by $\tau_{I I}^{\prime}(Z)=\sigma_{I I}$. The Hamiltonian formulation for PII is given explicitly as

$$
\begin{aligned}
& \frac{d q}{d Z}=\frac{\partial \sigma_{I I}}{\partial p}, \quad \frac{d p}{d Z}=-\frac{\partial \sigma_{I I}}{\partial q}, \\
& \sigma_{I I}(p, q, Z)=\frac{p^{2}}{2}+p\left(q^{2}+\frac{Z}{2}\right)+2 \gamma^{1 / 2} q .
\end{aligned}
$$

After eliminating $p(Z)$ from the first order system given by equation (3.19), one obtains PII for the function $q(Z)$. It is also clear from (3.19) that $p(Z)$ depends linearly on $q^{\prime}(Z)$, so that $\sigma_{I I}(Z)$ is a polynomial in $q(Z)$ and $q^{\prime}(Z)$ with a quadratic dependence on $q^{\prime}(Z)$. In summary, starting from a solution of PII one constructs $\sigma_{I I}(Z)$ which (after a change of variable) provides a solution $h(z)$ of equation (3.17). It is then possible to express the amplitude components $r_{j}$ of CNLS, in terms of solutions of the linear equation (3.16) whose coefficients $g(z)=h^{\prime}(z)$ and $g^{\prime}(z)=h^{\prime \prime}(z)$ depend on PII and its derivatives.

Case 2. Scaling reduction: In this case we set

$$
u_{j}(x, t)=t^{(-1+i \mu) / 2} q_{j}(z), \quad j=1,2, \quad z=x t^{-1 / 2}
$$

in analogy with the scaling reduction of the NLS equation (cf. equation (3.7)). Now each component $q_{j}(z)$ satisfies

$$
i q_{j}{ }^{\prime \prime}=z q_{j}{ }^{\prime}+q_{j}-i\left[\mu+2 s\left(\left|q_{1}\right|^{2}+\left|q_{2}\right|^{2}\right)\right] q_{j}, \quad j=1,2 .
$$

Next we introduce the variables:

$$
b_{j}=-i q_{j}{ }^{\prime}+z q_{j}, \quad j=1,2 \quad \text { and } \quad 2 a=i\left(\mu+2 s\left(\left|q_{1}\right|^{2}+\left|q_{2}\right|^{2}\right)\right.
$$

which satisfy a system of first order ODEs

$$
b_{j}^{\prime}=2 a q_{j}, \quad \bar{b}_{j}^{\prime}=-2 a \bar{q}_{j}, \quad a^{\prime}=s \sum_{j=1}^{2}\left(q_{j} \bar{b}_{j}-\bar{q}_{j} b_{j}\right)
$$

derived from equation (3.8) and its complex conjugate. The system (3.21) admits a first integral given by,

$$
a^{2}-s \sum_{j=1}^{2}|b|^{2}=-m^{2}
$$


$m$ being a constant. We again note that the scaling reduction can also be extended to the general case of multi-component $n$-CNLS equation in an obvious manner by setting $j=1,2, \ldots, n, n>2$ in equations (3.20)-(3.22).

In the following we show how to relate the function $a(z)$ to a solution of PIV. To this end, we differentiate the equation for $a(z)$ in (3.21) twice, use the remaining equations in (3.21) and the first integral in (3.22) to obtain a third order ODE for $a(z)$. This ODE for $a(z)$ can be integrated once by introducing the variable $f(z)$ where $f^{\prime}(z)=a(z)$. The final result is a third order ODE in $f(z)$ given by

$$
f^{\prime \prime \prime}=6 i f^{\prime 2}-z\left(z f^{\prime}-f\right)+2 \mu f^{\prime}+C z+2 i m^{2},
$$

where $C$ is a constant of integration. The change of variables

$$
\sigma_{I V}=-i k(f+C), \quad Z=z / k, \quad k^{2}=2 i
$$

in (3.23) leads to the equation for the Hamiltonian function $\sigma_{I V}$ associated with PIV, namely,

$$
\frac{d^{3} \sigma_{I V}}{d Z^{3}}=-6\left(\frac{d \sigma_{I V}}{d Z}\right)^{2}+4 Z\left(Z \frac{d \sigma_{I V}}{d Z}-\sigma_{I V}\right)+4 i \mu \frac{d \sigma_{I V}}{d Z}-8 m^{2}
$$

Moreover, there is a first integral of second order and second degree similar to the previous case of PII reduction (cf. equation (3.18), given by

$$
\left(\frac{d^{2} \sigma_{I V}}{d Z^{2}}\right)^{2}=4\left(Z \frac{d \sigma_{I V}}{d Z}-\sigma_{I V}\right)^{2}-4\left(\frac{d \sigma_{I V}}{d Z}-\nu_{0}\right)\left(\frac{d \sigma_{I V}}{d Z}-\nu_{1}\right)\left(\frac{d \sigma_{I V}}{d Z}-\nu_{2}\right)
$$

where one of the constants $\nu_{0}, \nu_{1}, \nu_{2}$ can be set to 0 without loss of generality. Equation 3.24b appears in Ref. [28] as well. The Hamiltonian formulation for PIV is described via the Poisson bracket relations

$$
\begin{aligned}
& \frac{d q}{d Z}=\left\{q, \sigma_{I V}\right\}, \quad \frac{d p}{d Z}=\left\{p, \sigma_{I V}\right\} \\
& \sigma_{I V}(q, p, Z)=\frac{2 p^{2}}{q}-p\left(q+2 Z+\frac{\nu_{1}}{q}\right)+\frac{\nu_{2}}{2} q
\end{aligned}
$$

where the fundamental Poisson bracket relation is given by

$$
\{p, q\}=-\{q, p\}=q .
$$

After eliminating $p(Z)$ from the first order system (3.25a), one finds that $q(Z)$ satisfies PIV. Note also that the PIV Hamiltonian $\sigma_{I V}$ is rational in $q(Z)$, but depends quadratically on $q^{\prime}(Z)$ as in the PII case. Consequently, $f(z)$ in (3.23) and in particular, the quantity $f^{\prime}(z)=a(z)=$ $i\left(\mu+2 s\left(\left|q_{1}\right|^{2}+\left|q_{2}\right|^{2}\right) / 2\right.$ in the reduced CNLS equation (3.20) are rational functions of the PIV transcendent and its derivatives. Therefore, equation (3.20) can be regarded as a second order linear equation for each $q_{j}$, with coefficients depending on PIV transcendent and its derivatives. Solutions of this linear ODE yield the the CNLS component fields $q_{j}$. 


\section{Conclusion}

In this paper we have studied systems of ODEs obtained from dimensional reductions of the CMB equations by scaling symmetry and of the CNLS equations by Galilean boost and scaling symmetries. We have derived new exact solutions of these ODEs and discussed their underlying Hamiltonian structures. The invariant solutions of the CMB equations are obtained in terms of the third Painlevé transcendent whereas, for the CNLS equations, the solutions invariant under Galilean boost and scaling are given in terms of the second and fourth Painlevé transcendents respectively. The obvious extensions of the reduction procedures to the multi-component $n$ CMB and $n$-CNLS equations are also indicated. In particular, for a Maxwell-Bloch system with probability density function $\left|a_{j}\right|^{2}$ for level $|j\rangle, j=1,2 \ldots, n, n \geq 1$, the ODE satisfied by the quantity $\left|a_{1}\right|^{2}+\left|a_{2}\right|^{2}+\ldots+\left|a_{n}\right|^{2}$ can be transformed to the general PIII equation with 2 arbitrary parameters. Similarly, for the reduced $n$-CNLS equations with complex fields $q_{j}, j=1,2 \ldots, n, n \geq 1$, the intensity function $I=\left|q_{1}\right|^{2}+\left|q_{2}\right|^{2}+\ldots+\left|q_{n}\right|^{2}$ turns out to be related to the Painlevé transcendents. The ODEs for $I$ obtained from Galilean boost and scaling symmetries can be transformed to the equations satisfied by the Hamiltonians $\sigma_{I I}$ and $\sigma_{I V}$ associated with PII and PIV respectively. We have also included a review of the known similarity reductions of scalar $(n=1)$ MB and NLS equations to Painlevé equations. These results provide a framework for comparison with the corresponding coupled systems. It is interesting to note that in all cases (considered in this work), the invariant solutions to the $n$ CMB and the $n$-CNLS equations are ultimately given independently of $n$, by the same Painlevé equation as the $n=1$ case. The only difference is that the Painlevé equations associated with the $n>1$ cases have more free parameters.

We conclude the paper on a slightly speculative note. It is possible to derive each of the Painlevé equations (PI-PVI) as the compatibility condition of a pair of $2 \times 2$ linear systems which arise in the study of isomonodromy deformation problems [28]. These linear systems are also obtained from the similarity reductions of $2 \times 2$ Lax pairs associated with equations solvable by IST (see e.g. [2]). Our study indicates that there are infinitely many linear systems $(n=1,2, \ldots)$ which arise from the Lax pairs associated with the $n$-CNLS and $n$-CMB equations and whose integrability conditions lead to the PII, PIII and PIV equations. It is conceivable then that each of the remaining Painlevé equations may also be derived from the isomonodromic deformations of not one, but a (denumerable) infinite number of pairs of linear equations. We hope to address this issue in the future.

\section{Acknowledgments}

S.C. is thankful to a London Mathematical Society travel grant and kind hospitality of Loughborough University where this work began. S.L.K. is grateful to Youngstown State University for his Sabbatical leave and for the financial support of a graduate research assistant, Victoria Vorotilkina. The helpful assistance of Victoria Vorotilkina is also acknowledged. R.H. thanks the Nuffield Foundation for partial support through grant number NAL/00344/G.

\section{References}

[1] Ablowitz M and Segur H 1981 Solitons and the Inverse Scattering Transform (Philadelphia: SIAM) 
[2] Ablowitz M and Clarkson P A 1991 Solitons, Nonlinear Evolution Equations and Inverse Scattering, LMS Lecture Note Series 149 (Cambridge: Cambridge University Press)

[3] Ablowitz M J, Ramani A and Segur H 1978 Lett. Nuevo Cim. 23 333-338

[4] Basharov A M, Maimistov A I and Manykin E A 1990 Sov. Phys. JETP 70 864-871

[5] Park Q-Han and Shin H J 1998 Phys. Rev. A 57 4643-4653

[6] Manakov S V 1974 Sov. Phys. JETP 38 248-253

[7] Zakharov V E and Schulman E I 1982 Physica D 4 270-274

[8] Sahadevan R, Tamizhmani K M and Lakshmanan M 1986 J. Phys. A: Math. Gen. 19 $1783-1791$

[9] Levi D, Menyuk C R and Winternitz P (Eds) 1994 Self-Similarity in Stimulated Raman Scattering (Montréal: CRM)

[10] McCall S L and Hahn E L 1969 Phys. Rev. 183 457-485

[11] Ablowitz M J, Kaup D J and Newell A C 1974 J. Math. Phys. 15 1852-1858

[12] Harris S E 194 Phys. Rev. Lett. 72 52-55

[13] Eberly J H, Pons M L and Haq H R 1994 Phys. Rev. Lett. 72 56-59

[14] Vemuri G and Vasavada K V 1996 Opt. Commun. 129 379-386

[15] Boller K J, Imamoglu A and Harris S E 1991 Phys. Rev. Lett. 66 2593-2596

[16] Harris S E 1989 Phys. Rev. Lett. 62 1033-1036

[17] Scully M O 1991 Phys. Rev. Lett. 67 1855-1858

[18] Park Q-Han and Shin H J 1998 Phys. Rev. A 57 4621-4642

[19] Chakravarty S and Ablowitz M J 1992 Painlevé Transcendents, Levi D and Winternitz P (Eds), (New York: Plenum Press), 331-343.

[20] Ince E L 1956 Ordinary Differential Equations (New York: Dover).

[21] Lukashevich N A 1967 Diff. Eqns. 3 994-999

[22] Baumann G, Glöckle W G and Nonnenmacher T F 1991 Proc. R. Soc. Lond. A 434 263-278

[23] Tajiri M 1983 J. Phys. Soc. Japan 52 1908-1917

[24] Chazy J 1911 Acta Math. 34 317-385

[25] Bureau F J 1972 Ann. di Math. 91 163-281

[26] Boiti M and Pempinelli F 1980 Nuovo Cim. B 59 40-58

[27] Gagnon L, Grammaticos B, Ramani A and Winternitz P 1989 J. Phys. A: Math. Gen. 22 499-509

[28] Jimbo M and Miwa T 1981 Physica D 2 407-448 